\title{
Rapid diagnosis of sputum negative miliary tuberculosis using the flexible fibreoptic bronchoscope
}

\author{
P A WILlCOX, P D POTGIETER, E D BATEMAN, S R BENATAR \\ From the Respiratory Clinic, Department of Medicine, University of Cape Town and Groote Schuur Hospital, \\ Observatory, Cape Town, South Africa
}

\begin{abstract}
Acid fast bacilli are seldom identified by direct staining of sputum smears in patients with miliary tuberculosis, so that delays in diagnosis are common. We report 41 patients with miliary tuberculosis who had negative sputum smears and who underwent bronchoscopy, bronchial brushing, and transbronchial biopsy. In two patients the procedure was repeated. A definitive diagnosis was obtained from bronchoscopy in 34 patients $(83 \%)$. Bronchial brushings yielded Mycobacterium tuberculosis in 24 of 42 bronchoscopies (57\%), 13 from direct smear and a further 11 from culture only. Transbronchial biopsies were diagnostic in 30 of 41 procedures (73\%), 28 from histological appearances, one from direct smear of the biopsy specimen, and another exclusively from culture. A rapid diagnosis was established in most patients (27/34), either by direct smear of brushings or biopsy specimens only (5), by histological examination only (14), or by both direct smear of brushings and histological examination (8). The diagnosis was confirmed later in a further seven patients by culture of brushings or specimens; in five of these non-caseating granulomas were initially found by histological examination. Fibreoptic bronchoscopy is a valuable technique for rapidly establishing the diagnosis of miliary tuberculosis.
\end{abstract}

In miliary tuberculosis sputum is often negative on direct smear ${ }^{1}$ and delays both in diagnosis and in starting treatment are common. There have been only a few reports on the use of the flexible fibreoptic bronchoscope in securing a prompt diagnosis in this condition and these have included only small numbers of patients. $^{2-5}$ We report the efficacy of bronchoscopy, bronchial brushing, and transbronchial biopsy in making a prompt diagnosis in 41 patients with miliary tuberculosis who had negative sputum smears.

\section{Methods}

Patients were suspected of having miliary tuberculosis if their chest radiographs showed the classic millet seed appearance with a diffuse infiltrate of nodules measuring less than $2 \mathrm{~mm}$ in diameter, ${ }^{6}$ but also (less commonly) if the nodules were unevenly distributed, irregular, and up to two to three times the size of the "millet seed."78

From April 1977 to June 198575 patients who were

Address for reprint requests: Dr PA Willcox, Respiratory Clinic, Groote Schuur Hospital, Observatory, Cape 7925, South Africa.

Accepted 1 April 1986 suspected of having miliary tuberculosis on radiographic and clinical grounds but whose sputum was negative on three or more direct smears, or who were unable to produce sputum, had fibreoptic bronchoscopy. Patients with a miliary pattern on the chest radiograph who had positive sputum on direct smear did not undergo bronchoscopy and are not included in this study. Similarly, patients with a miliary pattern on the chest radiograph in whom the diagnosis was initially confirmed by examination of cerebrospinal fluid, urine, or biopsy specimens of organs other than the lung did not undergo bronchoscopy. Details of the bronchoscopic technique and the handling of the samples have been described elsewhere. ${ }^{9}$ Transbronchial biopsy specimens were taken under radiographic screening in most cases to lessen the risk of pneumothorax. At least five biopsy specimens were obtained from only one lung. The specimens were placed in $10 \%$ formal saline and examined by haematoxylin and eosin and Ziehl-Neelsen stains. In 16 out of $43(37 \%)$ procedures a biopsy specimen was submitted for mycobacterial culture. Bronchial brushings were placed in normal saline, examined after ZiehlNeelsen staining, and cultured for Mycobacterium tuberculosis. Two patients underwent bronchoscopy on two occasions. 


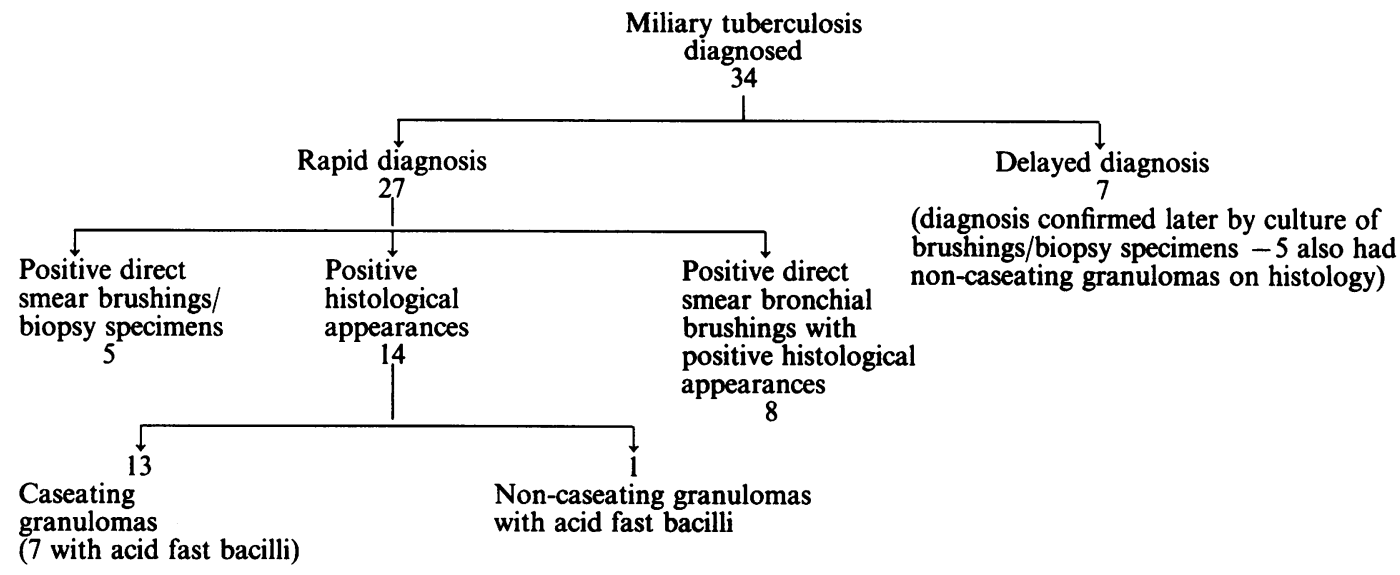

Analysis of the diagnostic sources in 34 patients.

Table 1 Histological findings in 28 transbronchial biopsy specimens confirming or suggesting tuberculosis

\begin{tabular}{lllll}
\hline & Caseating granulomas & Non-caseating granulomas & Chronic inflammatory changes \\
\hline Acid fast bacilli found & 7 & 3 & 1 & 11 \\
No acid fast bacilli found & 9 & 8 & -1 & 17 \\
Total & 16 & 11 & 18 \\
\hline
\end{tabular}

The criteria for the diagnosis of miliary tuberculosis in this setting were as follows: $(a)$ caseating or non-caseating granulomas or chronic inflammatory changes associated with the presence of acid fast bacilli or (b) granulomas (either caseating or noncaseating) without visible acid fast bacilli but the diagnosis supported by the finding of mycobacteria in bronchial brushings, or culture of biopsy specimens; by necropsy evidence of miliary tuberculosis; by culture of mycobacteria from cerebrospinal fluid, or by an appropriate response to antituberculous treatment.

\section{Results}

Of the 75 patients bronchoscoped, 41 were ultimately diagnosed as having miliary tuberculosis. Forty three procedures were performed on these 41 patients. Their ages ranged from 17 to 81 years, with a mean of 46 years. There were 22 women and 19 men. Twenty three patients were of mixed race, 13 black and five white.

A definitive diagnosis of miliary tuberculosis was made from the specimens obtained in 34 patients $(83 \%)$. The figure shows the results from the various specimens in these patients. A rapid diagnosis was established in the majority $(27 / 34$ ie $79 \%)$ either by positive direct smear of brushings/biopsy only (5), histology exclusively (14), or by both positive direct smear of brushings and histology (8). A delayed diagnosis was achieved in a further seven patients by culture of brushings or biopsies. In five of these patients non-caseating granulomas were present in the lung biopsies.

Bronchial brushings were obtained in 42 and transbronchial biopsy specimens in 41 of the 43 procedures. Brushings yielded Mycobacterium tuberculosis in 24 bronchoscopies $(57 \%), 13$ on direct smear and a further 11 on culture only. The histological features that confirmed or suggested the diagnosis in 28 transbronchial biopsy specimens are shown in table 1 . In two further cases the biopsy proved diagnostic, one with a positive direct smear and the other with a positive culture. In the 17 patients whose biopsy material contained granulomas (nine caseating and eight non-caseating) but no acid fast bacilli, bronchial brushings yielded a positive diagnosis in nine (four on direct smear and five on culture only). In the eight patients in whom granulomas were found without acid fast bacilli demonstrable in the biopsy specimens or in brushings there was clearing of the chest radiograph on treatment in six; one patient died (necropsy confirming miliary tuberculosis) and acid fast bacilli were subsequently cultured from the cerebrospinal fluid in another. Overall, acid fast bacilli were identified in 28 of the 34 patients and the 
Table 2 Histological diagnoses in 34 patients in whom miliary tuberculosis was excluded

Hut dwellers' anthracosis or silicoanthracosis
Sarcoidosis
Malignancy
Choriocarcinoma
Thyroid carcinoma
Pancreatic carcinoma
Unspecified granulomatous disorder

diagnosis was based on the presence only of caseating granulomas in five patients and of non-caseating granulomas in one.

Eight of 43 bronchoscopies (19\%), including two procedures on one patient, failed to provide diagnostic information. The diagnosis in these seven patients was made subsequently from smear or culture of sputum or specimens from other sites.

Miliary tuberculosis was excluded in 34 of the 75 patients with a miliary type chest radiograph and table 2 shows the subsequent diagnoses in these patients. Hut dwellers' anthracosis or silicoanthracosis, which was the most common disease to cause diagnostic difficulty, is a condition recognised in Southern Africa in people who have lived most of their lives in a rural environment. Transbronchial biopsy material shows evidence of heavy anthracosis with or without fibrosis and refractile material indicative of silica exposure. Follow up of those patients in whom no evidence of tuberculosis was found at bronchoscopy was consistent with this diagnosis. Similarly, in those cases where non-caseating granulomas were found in biopsy material and no bacteriological evidence of tuberculosis was found, the subsequent investigations and natural history confirmed the diagnosis of sarcoidosis.

The complication rate of bronchoscopy and transbronchial biopsy was low $(9 \%)$, with one moderate haemoptysis, two pneumothoraces (both requiring tube drainage), and transient hypoglycaemia in a diabetic patient.

\section{Discussion}

Miliary tuberculosis is characterised by diffuse haematogenous dissemination of Mycobacterium tuberculosis to many organs. ${ }^{6}$ The clinical presentation is very variable, ranging from a rapidly progressive condition to a chronic, insidious pyrexial illness. ${ }^{10}$ In countries where the incidence of tuberculosis has declined the pattern of miliary tuberculosis has changed; whereas in the past it was usually a complication of primary childhood infection, today it is mainly seen in the older age groups. ${ }^{6}$ Unfortunately the diagnosis is often missed in life and may be made only at necropsy, ${ }^{11-15}$ even in countries where the disease is common and the index of suspicion should be high. ${ }^{10}$ Delay in diagnosis, in part due to difficulty in obtaining bacteriological confirmation of the diagnosis from direct smears of sputum, ${ }^{1}$ is associated with an increased mortality rate. $^{810}$ Munt $^{1}$ reported positive sputum smears in $31 \%$ of 69 patients, and with subsequent cultures the diagnostic yield increased to $69 \%$. The time delay, however, makes culture helpful only in retrospect. In the past it was recommended that biopsy specimens be taken from organs more readily accessible than the lung. In a small series ${ }^{1}$ of nine liver biopsies, granulomas were found in six cases $(67 \%)$ but acid fast bacilli were found in only three. Buckingham ${ }^{16}$ reported liver granulomas in $34 \%$ of 32 patients with miliary tuberculosis but did not comment on the presence of acid fast bacilli. Granulomas in the liver are, however, non-specific unless associated with positive bacteriological findings. ${ }^{1718}$ The diagnosis of disseminated tuberculosis may be made from bone marrow biopsy specimens in $33 \%^{1}$ to $56 \%^{19}$ of cases. In Munt's series ${ }^{1}$ although 10 out of 30 patients had granulomas in their biopsy specimens, in only six of these cases were acid fast bacilli seen. Similarly, in Heinle's series of nine patients five had granulomas found on biopsy; acid fast bacilli were seen in one and four yielded positive cultures. ${ }^{19}$

Since many other diseases can cause a miliary infiltrate on the chest radiograph, ${ }^{20}$ there is a need for a highly sensitive, rapid diagnostic procedure for patients with miliary tuberculosis who have negative sputum smears. In the present series of 43 bronchoscopies in $\mathbf{4 1}$ patients, the overall diagnostic yield was $83 \%$ and a rapid diagnosis was established in most patients $(79 \%)$. There have been only a few previous reports on the value of bronchoscopy in securing a prompt diagnosis in these circumstances and these have been restricted to small numbers of patients. ${ }^{2-5}$ Sahn and Levin ${ }^{2}$ originally reported its value in 1975 in a single patient in whom transbronchial biopsy yielded numerous acid fast bacilli. Burk et $a l^{3}$ subsequently reported a diagnostic rate of $75 \%$ in eight patients with miliary tuberculosis. In two patients, however, sputum was positive on direct smear and in only two were granulomas and acid fast bacilli found. None of the smears of bronchial washings were positive on direct staining, and in the remainder cultures were negative. We have previously reported the use of fibreoptic bronchoscopy in the evaluation of 89 patients with sputum smear negative pulmonary tuberculosis, including 10 with miliary tuberculosis. ${ }^{5}$

The data reported in this larger series confirm our previous observation that fibreoptic bronchoscopy is a useful technique in safely and rapidly establishing the diagnosis of miliary tuberculosis when sputum is 
negative on direct smear. This allows appropriate treatment to be started with confidence in those patients in whom the diagnosis is confirmed on direct smear or by histological examination, or both. In the remainder the decision whether to treat immediately or to await the results of culture is dependent on clinical considerations.

We would like to acknowledge the contributions of the departments of pathology and microbiology and also the assistance of Sister C Wilson.

\section{References}

1 Munt RW. Miliary tuberculosis in the chemotherapy era: with a clinical review in 69 American adults. Medicine (Baltimore) 1972;51:139-55.

2 Sahn SA, Levin DC. Diagnosis of miliary tuberculosis by transbronchial lung biopsy. Br Med J 1975;ii:667-8.

3 Burk JR, Viroslav J, Bynum LJ. Miliary tuberculosis diagnosed by fibreoptic bronchoscopy and transbronchial biopsy. Tubercle 1978;59:107-9.

4 Wallace JM, Deutsch AL, Harrell JH, Moser KM. Bronchoscopy and transbronchial biopsy in evaluation of patients with suspected active tuberculosis. Am J Med 1981;70:1189-94.

5 Willcox PA, Benatar SR, Potgieter PD. Use of the flexible fibreoptic bronchoscope in diagnosis of sputum-negative pulmonary tuberculosis. Thorax 1982;37:598-601.

6 Sahn SA, Neff TA. Miliary tuberculosis. Am J Med 1974;56:495-505.

7 Crofton J, Douglas A. Respiratory diseases. 3rd ed. Oxford: Blackwell Scientific Publications, 1981.
8 Grieco $\mathrm{MH}, \mathrm{Chmel} \mathrm{H}$. Acute disseminated tuberculosis as a diagnostic problem: a clinical study based on twenty-eight cases. Am Rev Respir Dis 1974; 109:554-60.

9 Willcox PA, Benatar SR, Potgieter PD, Ferguson AD, Bateman ED. Fibreoptic bronchoscopy-Groote Schuur Hospital experience. $S$ Afr Med $J$ 1981;60:651-4.

10 Prout S, Benatar SR. Disseminated tuberculosis: a study of 62 cases. $S$ Afr Med J 1980;58:835-42.

11 Jacques J, Sloan JM. The changing pattern of miliary tuberculosis. Thorax 1970;25:237-40.

12 Proudfoot AT, Akhtar AJ, Douglas AC, Horne NW. Miliary tuberculosis in adults. Br Med J 1969;ii:273-6.

13 Rosenthal T, Pitlik S, Michaeli D. Fatal undiagnosed tuberculosis in hospitalised patients. $J$ Infect Dis 1975;131 (suppl): S51-6.

14 Treip C, Meyers D. Fatal tuberculosis in a General Hospital: a diagnostic problem. Lancet 1959;i:164-7.

15 Biehl JP. Miliary tuberculosis: a review of sixty-eight adult patients admitted to a municipal general hospital. Am Rev Respir Dis 1958;77:605-22.

16 Buckingham WB, Turner GC, Knapp WB, Young QD, Schaffner F. Liver biopsy in a tuberculosis hospital. Dis Chest 1956;29:675-83.

17 Gilinsky NH, Campbell JAC, Kirsch RE. The clinical spectrum of hepatic granuloma. $S$ Afr Med $J$ 1981;60:691-4.

18 Klatskin G. Hepatic granulomata: Problems in interpretation. Ann NY Acad Sci 1976;278:427-32.

19 Heinle EW, Jensen WN, Westerman MP. Diagnostic usefulness of marrow biopsy in disseminated tuberculosis. Am Rev Respir Dis 1965;91:701-5.

20 Turner-Warwick M, Strickland B. Diagnosis of diffuse disorders of the lung. In: Emerson P, ed. Thoracic medicine. London: Butterworth, 1981:857. 